\title{
Adaptive Finite-time Fuzzy Control of Nonlinear Active Suspension Systems With Input Delay
}

\author{
Jing Na, Member, IEEE, Yingbo Huang, Student Member, IEEE, Xing Wu, Shun-Feng Su, Fellow, IEEE, \\ and Guang Li, Member, IEEE
}

\begin{abstract}
This paper presents a new adaptive fuzzy control scheme for active suspension systems subject to control input time delay and unknown nonlinear dynamics. First, a predictor based compensation scheme is constructed to address the effect of input delay in the closed-loop system. Then, a fuzzy logic system (FLS) is employed as the function approximator to address the unknown nonlinearities. Finally, to enhance the transient suspension response, a novel parameter estimation error based finite-time (FT) adaptive algorithm is developed to online update the unknown FLS weights, which differs from traditional estimation methods, e.g. gradient algorithm with $e$-modification or $\sigma$-modification. In this framework, both the suspension and estimation errors can achieve convergence in finite-time. A Lyapunov-Krasovskii functional is constructed to prove the closed-loop system stability. Comparative simulation results based on a dynamic simulator built in a professional vehicle simulation software, Carsim, are provided to demonstrate the validity of the proposed control approach, and show its effectiveness to operate active suspension systems safely and reliably in various road conditions.
\end{abstract}

Index Terms-Fuzzy logic systems, Adaptive control, Active suspension systems, Input time delay, Finite-time convergence.

\section{INTRODUCTION}

$\mathbf{T}$ HE rapid development of automotive industry imposes more stringent requirements on the ride comfort and driving safety. In modern vehicle systems, suspension systems, as one of the most important vehicle chassis components, mainly affect the ride comfort, vehicle manoeuvrability and safety of drivers and occupants. Typically, suspension systems can be grouped into three categories: passive suspension systems, semi-active suspension systems and active suspension systems. Unlike passive and semi-active suspension systems, active suspension systems are equipped with extra actuation devices to provide or dissipate energy induced into the systems, thereby eliminating the vibrations and/or shocks transmitted to the vehicle body from irregular road roughness. In this

This work was supported by the National Natural Science Foundation of China under Grant 61873115, 61573174, and a joint PhD Scholarship between Chinese Scholarship Council (CSC) and Queen Mary University of London under Grant 201708530252. (Corresponding author: Yingbo Huang)

Jing $\mathrm{Na}$ and Xing Wu are with the Faculty of Mechanical and Electrical Engineering, Kunming University of Science and technology, Kunming, 650500, China (e-mail: najing25@163.com; xingwu@aliyun.com).

Yingbo Huang is with the Faculty of Mechanical and Electrical Engineering, Kunming University of Science and technology, Kunming, 650500, China, and also with the School of Engineering and Materials Science, Queen Mary University of London E1 4NS, UK (e-mail: Yingbo_Huang@126.com).

Shun-Feng Su is with the Department of Electrical Engineering, National Taiwan University of Science and Technology, Taipei, Taiwan (e-mail: sfsu@mail.ntust.edu.tw).

Guang $\mathrm{Li}$ is with the School of Engineering and Materials Science, Queen Mary University of London E1 4NS, UK (e-mail: g.li@qmul.ac.uk). respect, active suspension systems have been recognized as a promising pathway to achieve better suspension response and thus greatly improve the ride comfort and driving safety. Therefore, active suspension system construction and the associated control designs have attracted significant attentions from both industrial and academic communities [1]-[7].

For active suspension systems, designing robust and efficient control strategies is vital to operate them safely and reliably in various road conditions. Hence, many advanced control methods have been proposed for active suspensions since the past decades, such as adaptive control [8], robust control [9], fuzzy control [10] and $H_{\infty}$ control [11], etc. However, in practical engineering applications, active suspension systems have inherent input time delay phenomenon induced by the adopted hydraulic or pneumatic actuation devices. This critical delay phenomenon can also be found extensively in many practical control systems such as process control systems, networked control, where the existence of input delay can dramatically degrade the system performance and even trigger instability [11]. In this sense, designing an appropriate control strategy to accommodate the effect of input time delay has always been a practically useful yet challenging task. To address this issue, Smith [12] first proposed a Smith Predictor (SP) for linear systems with input delay by introducing a predictor structure to successfully avoid the appearance of delay in the denominator in the closed-loop transfer function. Aström [13] further tailored the SP framework for integral systems with input delay. In [14], Annaswamy modified the original SP method and presented an adaptive posicast controller (APC) to address the spark ignition engine speed control problem. Pade approximation approach was also adopted in [15] to approximate and then compensate the effect of small input delay in the control system. Although elegant mathematical analysis has been provided in these above mentioned control techniques for systems with input delay, they are mainly suitable for linear systems only. Specifically, extension of SP to nonlinear systems has still not been fully solved. Hence, control design for nonlinear systems with unknown dynamics and input delay is remaining as an open problem, in particular for vehicle suspension systems.

Very recently, Fischer and Dixon developed a saturated tracking controller [16] for uncertain nonlinear systems with input delay. This idea has been further explored for nonlinear systems with time-varying delays in the subsequent work [17], [18]. In this framework, a constructive predictor is proposed to address the effect of input delay, such that it can guarantee the uniform ultimate boundedness of the tracking error even in the 
presence of input time delay and uncertain dynamics. Hence, this paper will further study the possibility of incorporating this new technique into adaptive control for uncertain active suspension systems with input time delay and nonlinearities.

Apart from input delay, the unknown dynamics and nonlinearities are also inevitable in active suspension systems. With respect to this point, adaptive control methods combined with function approximators such as neural network (NN) and fuzzy logic system (FLS) have been extensively studied [19]-[27]. Among the aforementioned approaches, it is worth mentioning that FLS is characterized as a powerful tool to cope with uncertain dynamics and unknown nonlinearities by using linguistic knowledge representation and the corresponding fuzzy rules. Hence, adaptive fuzzy control techniques have been widely investigated for various plants [28]-[33]. In the FLS based adaptive control framework, the unknown weights of FLS are online updated by using gradient based algorithm to minimize the control error, then the convergence of the FLS weights cannot be guaranteed. However, it is well known that the approximation capability of FLS and the control response heavily rely on the estimation accuracy of these unknown weights. Hence, although much progress has been achieved in the adaptive fuzzy control designs, there still exist several issues that need to be further addressed. In particular, the conventional parameter estimation algorithms (e.g. $e$-modification or $\sigma$-modification) contain a damping term which prevents the estimated FLS weights from converging to their ideal values, which in turn lead to sluggish transient control response. According to the analysis in [34] and [35], the overall adaptive control performance can be improved provided that the estimated parameters can achieve convergence faster and smoother, with shorter settling time and smaller overshoot. To this end, it deserves further investigation to develop new learning algorithm for parameter estimation as [36]-[38] to further improve the adaptive control performance.

Inspired by the previous discussions, we will present a new adaptive fuzzy control design for active suspension systems with input time delay and unknown nonlinearities. We first introduce an input time delay compensation strategy in the adaptive control design, such that the effect of input delay on the closed-loop control system can be remedied. Then, a FLS is adopted to accommodate the uncertainties and nonlinearities. To guarantee better estimation of unknown FLS weights and thus improve the control response, a new parameter estimation algorithm containing information of the derived parameter error is developed to online update the FLS weights. This can be obtained by designing a set of auxiliary filtered matrices on the measurable system dynamics to derive a new leakage term, which can be superimposed on the gradient algorithm. In this new parameter estimation framework, accurate and finite-time convergence properties of the estimated FLS weights can be guaranteed. A LyapunovKrasovskii functional is constructed to prove the stability and convergence of the controlled system. A dynamic simulator is built by using a professional vehicle simulation software, Carsim 8.1, together with Matlab/Simulink, and comparative simulations are given to show the efficacy of the proposed approach.
The structure of this paper is organized as follow: Modeling of vehicle active suspension system and preliminary knowledge are given in Section II. The proposed input time delay compensation strategy, adaptive fuzzy control and the stability analysis are presented in Section III. Section IV provides comparative simulation results. Conclusions are given in V.

\section{System Modeling And Preliminaries}

\section{A. Modeling of Vehicle Active Suspension System}

A quarter-car active suspension system with uncertain dynamics and control input time delay is studied in this paper, whose schematic is given in Fig.1. The variables in Fig.1 are defined as: $m_{s}$ and $m_{u}$ represent the sprung mass and unsprung mass, which denote the vehicle chassis and the mass of tire assembly. $F_{d}$ is the damper force and $F_{s}$ is the spring force. $F_{t}$ and $F_{b}$ refer to the elasticity and damping forces of the tire. $z_{s}$ and $z_{u}$ are the vertical displacements of the sprung and unsprung masses, respectively. $z_{r}$ is the road excitation displacement and $U$ is the control input of the active suspension system. $\tau$ is a bounded positive constant input delay, which is induced by the adopted actuator.

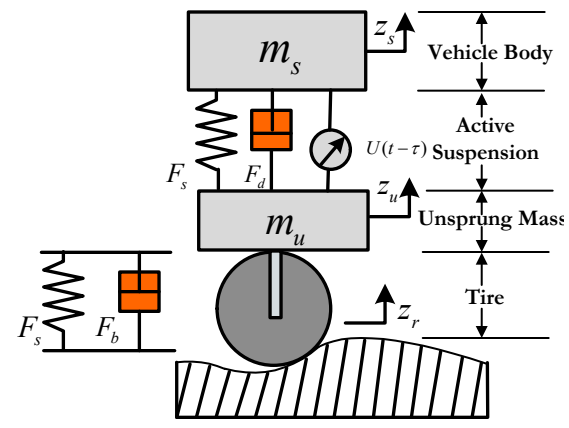

Fig. 1. Schematic representation of quarter-car active suspension systems

The differential equations for describing the studied quartercar active suspension system's motion behavior can be established based on the Newton's law as:

$$
\left\{\begin{array}{c}
m_{s} \ddot{z}_{s}+F_{d}\left(\dot{z}_{s}, \dot{z}_{u}\right)+F_{s}\left(z_{s}, z_{u}\right)=U(t-\tau) \\
m_{u} \ddot{z}_{u}-F_{d}\left(\dot{z}_{s}, \dot{z}_{u}\right)-F_{s}\left(z_{s}, z_{u}\right)+F_{t}\left(z_{u}, z_{r}\right) \\
+F_{b}\left(\dot{z}_{u}, \dot{z}_{r}\right)=-U(t-\tau)
\end{array}\right.
$$

To facilitate the control design, we set the system state variables as:

$$
x_{1}=z_{s}, x_{2}=\dot{z}_{s}, x_{3}=z_{u}, x_{4}=\dot{z}_{u}
$$

Then system (1) can be rewritten in a state-space form as:

$$
\left\{\begin{aligned}
\dot{x}_{1} & =x_{2} \\
\dot{x}_{2} & =\frac{1}{m_{s}}\left(-F_{d}\left(x_{2}, x_{4}\right)-F_{s}\left(x_{1}, x_{3}\right)+U_{\tau}\right) \\
\dot{x}_{3} & =x_{4} \\
\dot{x}_{4} & =\frac{1}{m_{u}}\left(F_{d}\left(x_{2}, x_{4}\right)+F_{s}\left(x_{1}, x_{3}\right)-F_{t}\left(x_{3}, z_{r}\right)\right. \\
& \left.-F_{b}\left(x_{4}, \dot{z}_{r}\right)-U_{\tau}\right)
\end{aligned}\right.
$$

where $U_{\tau}=U(t-\tau)$ defines the delayed control input signal. 
Remark 1: Unlike most existing control schemes for active suspensions (e.g. [3], [5], [8]), the input delay induced by the actuator dynamics is considered in the vehicle system and the subsequent control synthesis in this paper. Moreover, the damper force $F_{d}$ and spring force $F_{s}$ are considered to be nonlinear, unknown rather than the unrealistically known, linear cases as in [5], [9], [11]. These advancements are not trivial since the derived model (3) can cover more realistic suspension system dynamics, while specific compensation of the input delay has to be considered.

Remark 2: In practical operation scenarios of vehicle suspension systems, the vertical displacement $x_{1}$, velocity $x_{2}$ and acceleration $\dot{x}_{2}$ can be measured by the configured signal acquisition devices, e.g. displacement/acceleration sensor. Hence, the system state variables $x_{1}, x_{2}$ and $\dot{x}_{2}$ in (3) are assumed to be measurable and will be used in the control implementation.

\section{B. Problem Formulation}

The aim of this paper is to develop a new adaptive control scheme for active suspension system (3) with unknown forces and input delay, such that the following suspension performance requirements can be addressed:

- Performance Requirement 1 (PR1)-Ride Comfort: The vertical displacement and acceleration of vehicle body should be minimized under external road disturbances, since these two variables are widely regarded as the key performance indices to evaluate the vehicle ride comfort. Hence, the proposed control should have capability of attenuating vibrations and/or shocks as much as possible and maintaining the stabilization of vehicle body. This requirement can be described by:

$$
\text { PR1: } \min \left(z_{s}\right) \quad \& \quad \min \left(\ddot{z}_{s}\right)
$$

- Performance Requirement 2 (PR2)-Road Holding and Deflection: In order to ensure the driving safety of drivers and occupants, the firm, continuous connection of the wheel with the road should be strictly guaranteed. This fact requires that the dynamic tire loads should not exceed the limitation, which can be described by:

$$
\text { PR2: } \frac{\left|F_{t}+F_{b}\right|}{\left(m_{s}+m_{u}\right) g}<1
$$

\section{- Performance Requirement 3 (PR3)-Suspension Stroke:}

Due to the restrictive mechanical active suspension structure, the proposed control should guarantee the suspension stroke within a reasonably allowable maximum suspension movement limitation $z_{\max }$, given by:

$$
\text { PR3: }\left|z_{s}-z_{u}\right|<z_{\max }
$$

\section{Function Approximation with FLS}

Fuzzy logic systems (FLS) have been widely used in the control designs for nonlinear systems because of its capability of approximating unknown nonlinear functions without the requirement of a priori accurate knowledge of system dynamics. The general structure of the FLS used for the function approximation is shown in Fig.2.

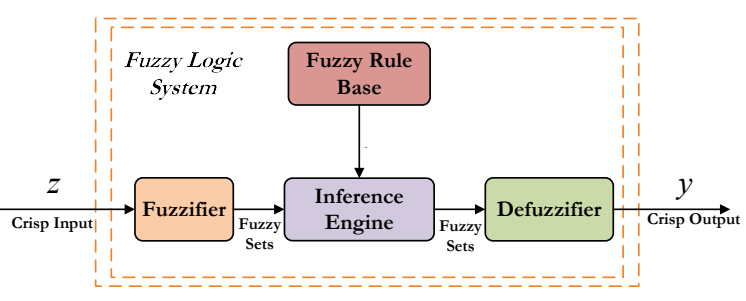

Fig. 2. Overview of FLS structure

In this paper, we will adopt a FLS to approximate a lumped unknown nonlinear function $F(x) \in \mathbb{R}^{m}$ with $x \in \mathbb{R}^{n}$ being the input variables, which stems from the unknown dynamics in the active suspension system given in Fig.1. The formulation of FLS is explained as follows [22]:

i) Fuzzifier: Fuzzifier provides a map from the crisp input $x$ to the fuzzy linguistic behavior with the following Gaussian function:

$$
\xi_{j}(x)=\exp \frac{\left(x-\varsigma_{j}\right)^{\mathrm{T}}\left(x-\varsigma_{j}\right)}{\sigma^{2}}
$$

where $\varsigma_{j}=\left[\varsigma_{1}, \varsigma_{2}, \ldots, \varsigma_{n}\right]^{\mathrm{T}} \in \mathbb{R}^{n}$ is the center vector placed on a regular lattice and $\sigma$ denotes the width of the Gaussian function.

ii) Fuzzy Rule Base: A fuzzy rule base is composed of various fuzzy rules in the form of IF-THEN statement, denoting relationship between the input set and output set using the Mamdani min-implication as:

Rule $j,(j=1,2, \ldots, \gamma)$ :

$$
\begin{gathered}
\text { IF } x_{i} \text { is } A_{j i}, i=1 \ldots n \\
\text { THEN } y_{o} \text { is } B_{j o}, o=1 \ldots m
\end{gathered}
$$

where $x=\left[x_{1} \ldots x_{n}\right] \in \mathbb{R}^{n}$ and $y=\left[y_{1} \ldots y_{m}\right] \in \mathbb{R}^{m}$ are the variables corresponding to the input and output, respectively. $A_{j i}$ and $B_{j o}$ are the fuzzy sets, and $\gamma$ is the number of the rules.

iii) Fuzzy Inference Engine and Defuzzifier: The FLS adopted here with the Gaussian fuzzifier, sum-product inference and centre-average defuzzifier is defined as:

$$
y_{o}(z)=\frac{\sum_{j=1}^{\gamma} \phi_{j o} \prod_{i=1}^{n} \mu A_{j i}\left(x_{i}\right)}{\sum_{j=1}^{\gamma} \prod_{i=1}^{n} \mu A_{j i}\left(x_{i}\right)}
$$

where $\phi_{j o}=\max _{y_{o}(x) \in \mathbb{R}}\left(\mu B_{j o}\left(\phi_{j}\right)\right)$ is the point at which $\mu B_{j o}\left(\phi_{j}\right)$ achieves its maximum value and $\mu A_{j i}\left(x_{i}\right)$ denotes the membership function. $\Phi(x)=$ $\left[\xi_{1}(x), \xi_{2}(x), \ldots, \xi_{\gamma}(x)\right]^{\mathrm{T}} \in \mathbb{R}^{\gamma}$, with $\xi_{j}(x)=$ $\prod_{i=1}^{k} \mu A_{j i}\left(x_{i}\right) / \sum_{j=1}^{\gamma} \prod_{i=1}^{k} \mu A_{j i}\left(x_{i}\right)$ the Gaussian function given in (7). Define $W_{o}=\left[\phi_{1 o}, \phi_{2 o}, \ldots, \phi_{\gamma o}\right]^{\mathrm{T}} \in \mathbb{R}^{\gamma}$ and $W=\left[W_{1}, W_{2}, \ldots, W_{m}\right] \in \mathbb{R}^{\gamma \times m}$. Hence, the FLS can be formulated as the following form:

$$
y(x)=W^{\mathrm{T}} \Phi(x)
$$

Since all the membership functions are chosen as the Gaussian membership function, the following lemma holds: 
Lemma 1: [39] Given a compact set $\Omega_{o}$, we define $f_{o}(z) \in$ $\mathbb{R}$ as a continuous function. Then, a FLS can be constructed such that:

$$
\sup _{f_{o}(x) \in \Omega_{o}}\left|f_{o}(x)-y_{o}(x)\right| \leq \vartheta
$$

with $y_{o}=W_{o}^{T} \Phi(x)$ is true for any small positive constant $\vartheta$.

Based on Lemma 1, any contiguous function vector $F(x)=$ $\left[f_{1}(x), f_{2}(x), \ldots, f_{m}(x)\right] \in \mathbb{R}^{m}$ can be expressed as:

$$
F(x)=W^{\mathrm{T}} \Phi(x)+\varepsilon
$$

where $W \in \mathbb{R}^{\gamma \times m}$ is the ideal FLS weights matrix, $\Phi \in \mathbb{R}^{\gamma}$ is the FLS regressor vector and $\varepsilon \in \mathbb{R}^{m}$ is the residual FLS approximation error, which are bounded by $\|W\| \leq W_{b}$ and $\|\varepsilon\| \leq \varepsilon_{b}$ for constants $W_{b}, \varepsilon_{b}>0$. It is noted that the FLS approximation error $\varepsilon$ can be set arbitrarily small by tuning the number of FLS rules $\gamma$ sufficiently large.

\section{Adaptive Active Suspensions Control Design AND ANALYSIS}

\section{A. Adaptive Finite-time Fuzzy Control}

As explained in the introduction, the input delay is a crucial and challenging problem in the control designs for nonlinear systems with uncertain dynamics. Although some input delay compensation strategies [11], [14], [15] have been proposed, only few work have been reported for nonlinear systems with input delay. On the other hand, nonlinear active suspension systems rather than linear counterparts in [5], [11], [15] are considered to cover more realistic applications. These two issues will be considered in this section, where we will present a novel finite-time convergence adaptive fuzzy control scheme to effectively address the uncertainties/nonlinearities and compensate the input delay. The diagram of the proposed control system can be found in Fig.3. Note that as clarified in Remark 2, the system states $x_{1}, x_{2}$ and $\dot{x}_{2}$ can be measured by commercial sensors (e.g. displacement/acceleration sensor) configured in the vehicle system, and thus they can be used in the control implementation.

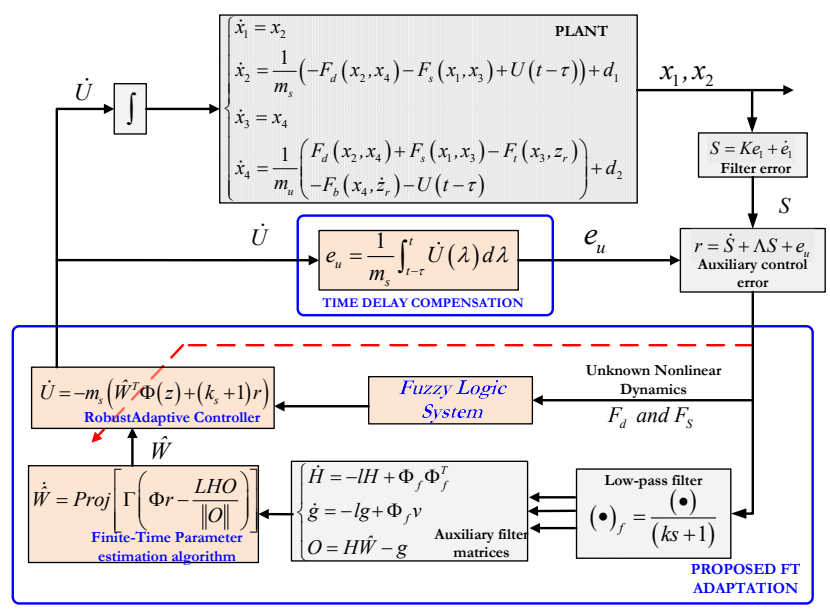

Fig. 3. Block diagram of the proposed control strategy.

Regarding to the primary requirement PR1, the proposed control should regulate the vehicle motion (i.e. $x_{1} \rightarrow 0$ ) as much as possible to mitigate the road excitation $z_{r}$. To accomplish the regulation of vertical displacement, a control error, $e_{1} \in \mathbb{R}$, to be minimized is defined as:

$$
e_{1}=x_{1}
$$

Moreover, a filter error $S \in \mathbb{R}$ is designed as:

$$
S=K e_{1}+\dot{e}_{1}
$$

where $K \in \mathbb{R}^{+}$is a positive constant.

To derive a delay-free predictor to compensate the effect of input delay in (1), an integral control input signal $e_{u} \in \mathbb{R}$ is introduced as inspired by [17], [18], which is designed based on the Leibniz-Newton formula:

$$
e_{u}=\frac{1}{m_{s}}\left(U-U_{\tau}\right)=\frac{1}{m_{s}} \int_{t-\tau}^{t} \dot{U}(\lambda) d \lambda
$$

To eliminate the input delay by incorporating the compensator $e_{u}$ into the control design, another error signal, $r \in \mathbb{R}$, is defined along (13) and (14) as:

$$
r=\dot{S}+\Lambda S+e_{u}
$$

where $\Lambda \in \mathbb{R}^{+}$is also a positive control constant. As mentioned before, the system states $x_{1}, x_{2}$ and $\dot{x}_{2}$ can be measured in the suspension system, such that the error signal $r$ can be used in the control implementation in this paper.

Then, the time derivative of $r$ can be deduced along (3) and (14) as:

$$
\begin{aligned}
\dot{r} & =\ddot{S}+\Lambda \dot{S}+\dot{e}_{u} \\
& =K \dot{x}_{2}+\frac{1}{m_{s}}\left(-\dot{F}_{d}\left(x_{2}, x_{4}\right)-\dot{F}_{s}\left(x_{1}, x_{3}\right)\right) \\
& +\frac{1}{m_{s}} \dot{U}_{\tau}+\Lambda \dot{S}+\frac{1}{m_{s}} \dot{U}-\frac{1}{m_{s}} \dot{U}_{\tau}
\end{aligned}
$$

where $F(x)=\frac{1}{m_{s}}\left(-\dot{F}_{d}\left(x_{2}, x_{4}\right)-\dot{F}_{s}\left(x_{1}, x_{3}\right)\right)$ can be defined as the lumped unknown system dynamics.

Assumption 1: The damper force $F_{d}$ and spring force $F_{s}$ are continuous on a compact set $\Omega$ and their first derivatives are bounded on the compact set.

Based on the analysis shown in [40], [41], we know that the derivative of unknown system dynamics is also a nonlinear function of the measured system states, such that the lumped unknown dynamics $F(x)$ can be approximated by a FLS shown in Lemma 1 as:

$$
\begin{aligned}
F(x) & =\frac{1}{m_{s}}\left(-\dot{F}_{d}\left(x_{2}, x_{4}\right)-\dot{F}_{s}\left(x_{1}, x_{3}\right)\right) \\
& =W^{\mathrm{T}} \Phi(x)+\varepsilon
\end{aligned}
$$

By using (17), then equation (16) can be reformulated as:

$$
\dot{r}=K \dot{x}_{2}+W^{\mathrm{T}} \Phi(x)+\Lambda \dot{S}+\frac{1}{m_{s}} \dot{U}+\varepsilon
$$

For the ease of subsequent analysis, we further define an auxiliary function $\tilde{N} \in \mathbb{R}$ as:

$$
\tilde{N}=K \dot{x}_{2}+\Lambda \dot{S}+S+r
$$

Then following the analysis procedure given in [17], [18] and [42], the Mean Value Theorem can be applied on this continuously differentiable function $\tilde{N}$. Hence, according to 
Remark 1 and Assumption 1, one can claim that the $\tilde{N}$ can yields: be upper bounded by:

$$
\|\tilde{N}\| \leq \zeta(\|z\|)\|z\|
$$

with $\zeta(\cdot)$ being a positive, strictly increasing function, and the coordinates $z$ given by:

$$
z=\left[e_{1}, S, e_{u}, r\right]^{\mathrm{T}}
$$

Therefore, equation (18) can be rewritten by using (19) as:

$$
\dot{r}=\tilde{N}+W^{\mathrm{T}} \Phi(x)+\varepsilon+\frac{1}{m_{s}} \dot{U}-S-r
$$

Now, a delay-free control input $U$ can be designed as:

$$
U=-m_{s} \int_{0}^{t}\left[\hat{W}^{\mathrm{T}}(\lambda) \Phi(x(\lambda))+\left(k_{s}+1\right) r(\lambda)\right] d \lambda
$$

where $k_{s}>0$ is the feedback gain, and $\hat{W}$ is the estimate of the unknown FLS weights $W$, which will be online updated by a new adaptive law with guaranteed finite-time convergence as presented in the next subsection.

Taking the derivative of $U$ given in (23) with respect to time $t$, then it follows:

$$
\dot{U}=-m_{s}\left(\hat{W}^{\mathrm{T}} \Phi(x)+\left(k_{s}+1\right) r\right)
$$

Substituting (24) into (22), the closed-loop system dynamics (22) can be reformulated as:

$$
\dot{r}=\tilde{N}+\tilde{W}^{\mathrm{T}} \Phi(x)+\varepsilon-\left(k_{s}+2\right) r-S
$$

where $\tilde{W}=W-\hat{W}$ is the estimation error of the FLS weights.

\section{B. Adaptive Law with Finite-Time Convergence}

To implement the adaptive fuzzy control (23), we need to design an adaptive law to obtain the estimated FLS weights $\hat{W}$ online. In most existing adaptive fuzzy control designs, the adaptive laws are usually designed based on the tracking error $r$ with $e$-modification or $\sigma$-modification. In these algorithms, the involved damping term prevents the estimated weights from converging to their ideal values. However, based on the certainty equivalence principle [34], if the estimated weights could converge to their ideal values fast and accurately, the overall control performance can be greatly improved. Specifically, as shown in (25), if the estimation error of the FLS weights $\tilde{W}$ could be driven to zero with adequately fast convergence rate, the effect of the undesired error $\tilde{W}^{\mathrm{T}} \Phi(x)$ on the convergence of $r$ can be eliminated, such that better transient control response can be obtained [43].

Following the above discussions, we will develop a novel adaptive parameter estimation algorithm which is able to guarantee that the estimated FLS weights converge to their ideal values (i.e. $\hat{W} \rightarrow W$ ) with guaranteed finite-time convergence.

To realize this objective, a low-pass filter $(\bullet)_{f}=$ $(\bullet) /(\kappa s+1)$ is applied on (22) to avoid the use of $\dot{r}$, which

$$
\begin{cases}\kappa \dot{r}_{f}+r_{f}=r, & r_{f}(0)=0 \\ \kappa \dot{\tilde{N}}_{f}+\tilde{N}_{f}=\tilde{N}, & \tilde{N}_{f}(0)=0 \\ \kappa \dot{S}_{f}+S_{f}=S, & S_{f}(0)=0 \\ \kappa \dot{\Phi}_{f}+\Phi_{f}=\Phi, & \Phi_{f}(0)=0 \\ \kappa \ddot{U}_{f}+\dot{U}_{f}=\dot{U}, & \dot{U}_{f}(0)=0\end{cases}
$$

where the filter coefficient $\kappa \in \mathbb{R}^{+}$should be set as a small positive constant to retain fast convergence of the filter.

Then, according to the first equation of (26) and (22), it can be derived:

$$
\dot{r}=\frac{r-r_{f}}{\kappa}=\tilde{N}_{f}+W^{\mathrm{T}} \Phi_{f}+\frac{1}{m_{s}} \dot{U}_{f}-S_{f}-r_{f}+\varepsilon_{f}
$$

where $\varepsilon_{f}$ is the filtered variable of the FLS approximation error $\varepsilon$.

Moreover, we design the following auxiliary filter matrices $\mathcal{H} \in \mathbb{R}^{\gamma \times \gamma}, \mathcal{G} \in \mathbb{R}^{\gamma \times 1}$ and $\mathcal{O} \in \mathbb{R}^{\gamma \times 1}$ as:

$$
\left\{\begin{aligned}
\dot{\mathcal{H}} & =-\ell \mathcal{H}+\Phi_{f} \Phi_{f}^{\mathrm{T}}, & & \mathcal{H}(0)=0 \\
\dot{\mathcal{G}} & =-\ell \mathcal{G}+\Phi_{f} \mathcal{V}, & & \mathcal{G}(0)=0 \\
\mathcal{O} & =\mathcal{H} \hat{W}-\mathcal{G} & &
\end{aligned}\right.
$$

where $\mathcal{V}=\left(r-r_{f}\right) / \kappa-\tilde{N}_{f}-\frac{1}{m_{s}} \dot{U}_{f}+S_{f}+r_{f}$, and $\ell \in \mathbb{R}$ is also a positive design parameter to guarantee the boundedness of $\mathcal{H}, \mathcal{G}$.

The solution of $\mathcal{H}$ and $\mathcal{G}$ can be calculated from (28) as:

$$
\left\{\begin{array}{l}
\mathcal{H}(t)=\int_{0}^{t} e^{-\ell(t-\lambda)} \Phi_{f}(\lambda) \Phi_{f}^{\mathrm{T}}(\lambda) d \lambda \\
\mathcal{G}(t)=\int_{0}^{t} e^{-\ell(t-\lambda)} \Phi_{f}(\lambda) \mathcal{V}(\lambda) d \lambda
\end{array}\right.
$$

From the definition of of $\mathcal{H}, \mathcal{G}$ and $\mathcal{O}$ given in (28) and (29), we have:

$$
\mathcal{O}=\mathcal{H} \hat{W}-\mathcal{G}=\mathcal{H} \hat{W}-\mathcal{H} W+\chi=-\mathcal{H} \tilde{W}+\chi
$$

where $\chi=-\int_{0}^{t} e^{-\ell(t-\lambda)} \Phi_{f}(\lambda) \varepsilon_{f}(\lambda) d \lambda$ denotes the residual error stemming from the inherent FLS approximation error, which is bounded, since the filtered FLS approximation error $\varepsilon_{f}$ and regressor vector $\Phi_{f}$ are all bounded. Therefore, we have that $\chi$ is bounded fulfilling the condition $\sup _{t \geq 0}\|\chi\| \leq \bar{\chi}$ for a positive constant $\bar{\chi}>0$.

It is shown in (30) that the online calculated vector $\mathcal{O}$ based on the derived variables $r, \Phi, S, U, \tilde{N}$ with filter operations given in (26) and (27) contains information of the estimation error $\tilde{W}$. Therefore, the use of this derived variable $\mathcal{O}$ in the adaptive law can enhance the performance of parameter estimation. Now, the following finite-time parameter estimation algorithm $\operatorname{Proj}(\cdot)$ with projection operation can be designed to online update the FLS weights:

$$
\dot{\hat{W}}=\Gamma \operatorname{Proj}(\Phi r-\mathcal{M})
$$

The novel leakage term $\mathcal{M}$ including a sliding mode type term with $\mathcal{O}$ to achieve finite-time convergence is given by

$$
\mathcal{M}=\mathcal{L O}+\frac{\mathcal{L} \mathcal{H O}}{\|\mathcal{O}\|}
$$


where $\Gamma \in \mathbb{R}$ and $\mathcal{L} \in \mathbb{R}$ are positive learning gains. $\operatorname{Proj}(\bullet)$ is a smooth projection operation such that the estimated FLS weights $\hat{W}$ can be always ensured within a convex set $\mathbb{E}$ as stated in [25].

The practical implementation of the proposed approach for the studied active suspension system (1) can be described as the following algorithm:

\section{Algorithm 1 Control Implementation for Active Suspension} System

Step 1: Initializing the fuzzy logic system topology and choosing proper membership functions;

Step 2: Measuring the system state $x_{i}$, choosing positive constants $K, \Lambda$ and feedback gain $k_{s}$ to design controller $U$ based on (23);

Step 3: Adjusting the learning gains $\mathcal{L}$ and $\Gamma$ for online updating unknown FLS weights $\hat{W}$ with the proposed adaptive algorithm (31);

Step 4: Employing the designed controller $U$ derived from (23) for the active suspension system (1).

Remark 3: The implementation of the above control (23) is straightforward as shown in (24). The first term from the FLS, $\hat{W}^{\mathrm{T}} \Phi$, is used to address the lumped unknown dynamics, and the second term is the error feedback calculated based on (15). To achieve satisfactory suspension performance and estimation convergence, the filter coefficients $\kappa, \ell$ should be set as small positive constants. Moreover, the feedback gain $k_{s}$, filter coefficients $K, \Lambda$, and adaptive learning gains $\Gamma, \mathcal{L}$ should be selected properly. In general, large gains $k_{s}, K, \Lambda$ contribute to improving the suspension error rate, while too large gains may make the system sensitive to noise and disturbances. Moreover, large learning gains $\Gamma$ and $\mathcal{L}$ can improve the parameter estimation convergence speed, but can result in oscillations of control actions.

Remark 4: In most existing adaptive fuzzy control literature, the traditional adaptive law (the first term of (31)) with $\sigma$ modification is used to update the FLS weights, which means that the estimation error $\tilde{W}$ and control error $r$ can be proved to be uniformly ultimately bounded only, while the transient control response may be poor due the sluggish convergence of this adaptive law. In this paper, a novel leakage term $\mathcal{M}$, containing the parameter estimation error information $\tilde{W}$, is imposed on the traditional adaptive law $\hat{W}=\Gamma \Phi r$ as in (31), which can improve the parameter estimation convergence and the control error. In particular, a sliding mode term given in (32) is used, such that the finite-time convergence of $\tilde{W}$ can be guaranteed as shown in next subsection.

\section{Closed-loop System Stability}

In this subsection, we will focus on the stability analysis of the proposed control system with control action (24) and finitetime parameter estimation algorithm (31). We first present the following Lemma:

Lemma 2 [38]: The matrix $\mathcal{H}$ defined in (28) is positive definite provided that the FLS regressor $\Phi$ given in (17) is persistently excited (PE). This fact implies that its minimum eigenvalue satisfies $\lambda_{\min }(\mathcal{H})>\varrho>0$ with $\varrho \in \mathbb{R}$ being a positive constant.

Now, the following theorem can be presented:

Theorem 1: Consider the active suspension system (3) with the proposed controller (24) and finite-time parameter estimation algorithm (31), then all signals in the closed-loop system are bounded, and the vertical displacement $x_{1}$ will exponentially converge to a small residual set around zero.

Proof: We choose a Lyapunov function $V$ as follows:

$$
V=V_{1}+V_{2}
$$

where the first term $V_{1}$ is selected as:

$$
V_{1}=\frac{1}{2} e_{1}^{2}+\frac{1}{2} S^{2}+\frac{1}{2} e_{u}^{2}+\frac{1}{2} r^{2}
$$

and the second term $V_{2}$ is designed as:

$$
V_{2}=\frac{1}{2} \tilde{W}^{\mathrm{T}} \Gamma^{-1} \tilde{W}+\mathcal{P}_{L K}+\mathcal{R}_{L K}
$$

where $\mathcal{P}_{L K}, \mathcal{R}_{L K} \in \mathbb{R}$ are the Lypunov-Krasovskii functionals, which are used to tackle the input delay. Hence, $\mathcal{P}_{L K}$ and $\mathcal{R}_{L K}$ are give as follows:

$$
\begin{aligned}
\mathcal{P}_{L K} & =\frac{1}{m_{s}} \int_{t-\tau}^{t}\|\dot{U}(\lambda)\|^{2} d \lambda \\
\mathcal{R}_{L K} & =\int_{t-\tau}^{t}\left(\int_{s}^{t}\|\dot{U}(\lambda)\|^{2} d \lambda\right) d s
\end{aligned}
$$

Based on (33), (34) and (35), the following inequality can be verified for the Lyapunov function $V$ :

$$
\frac{1}{2}\|\mathcal{Y}\|^{2} \leq V(\mathcal{Y}) \leq\|\mathcal{Y}\|^{2}
$$

where $\mathcal{Y}=\left[z, \tilde{W}, \sqrt{\mathcal{P}_{L K}}, \sqrt{\mathcal{R}_{L K}}\right]$

The time derivative of $V_{1}$ can be calculated as:

$$
\begin{aligned}
\dot{V}_{1} & =e_{1} \dot{e}_{1}+S \dot{S}+e_{u} \dot{e}_{u}+r \dot{r} \\
& =e_{1}\left(S-K e_{1}\right)+S\left(r-\Lambda S-e_{u}\right)+\frac{e_{u}}{m_{s}}\left(\dot{U}-\dot{U}_{\tau}\right) \\
& +r \tilde{N}+r \varepsilon+r \tilde{W}^{\mathrm{T}} \Phi-\left(k_{s}+2\right) r^{2}-r S
\end{aligned}
$$

By using the Young's inequality, the following inequalities can be obtained:

$$
\begin{aligned}
e_{1} S \leq \frac{1}{2} e_{1}^{2} & +\frac{1}{2} S^{2}, \quad e_{1} r \leq \frac{1}{2} e_{1}^{2}+\frac{1}{2} r^{2} \\
-S e_{u} & \leq \frac{1}{2} S^{2}+\frac{1}{2} e_{u}^{2} \\
e_{u}\left(\dot{U}-\dot{U}_{\tau}\right) & \leq \frac{1}{2}\left\|e_{u}\right\|^{2}+\frac{1}{2}\left\|\dot{U}-\dot{U}_{\tau}\right\|^{2} \\
\left\|\dot{U}-\dot{U}_{\tau}\right\|^{2} & \leq 2\|\dot{U}\|+2\left\|\dot{U}_{\tau}\right\|^{2}
\end{aligned}
$$

Then, equation (38) can be reformulated by using (39) as:

$$
\begin{aligned}
\dot{V}_{1} \leq & -\left(K-\frac{1}{2}\right) e_{1}^{2}-(\Lambda-1) S^{2}+\frac{1}{2}\left(1+\frac{1}{m_{s}}\right)\left\|e_{u}\right\|^{2} \\
& -\left(k_{s}+1\right) r^{2}+\frac{1}{2} \zeta^{2}(\|z\|)\|z\|^{2}+\frac{1}{2} \varepsilon_{b}^{2} \\
& +\tilde{W}^{\mathrm{T}} \Phi r+\frac{1}{m_{s}}\|\dot{U}\|^{2}+\frac{1}{m_{s}}\left\|\dot{U}_{\tau}\right\|^{2}
\end{aligned}
$$


By using the Leibniz-Newton Rule, the derivative of $\mathcal{P}_{L K}$ and $\mathcal{R}_{L K}$ with respect to time $t$ can be deduced as:

$$
\begin{aligned}
\dot{\mathcal{P}}_{L K} & =\frac{1}{m_{s}}\|\dot{U}\|^{2}-\frac{1}{m_{s}}\left\|\dot{U}_{\tau}\right\|^{2} \\
\dot{\mathcal{R}}_{L K} & =\tau\|\dot{U}\|^{2}-\int_{t-\tau}^{t}\|\dot{U}(\lambda)\|^{2} d \lambda
\end{aligned}
$$

Hence, the time derivative of $V_{2}$ can be obtained along with (35) and (41a)-(41b) as:

$$
\begin{aligned}
\dot{V}_{2}= & -\tilde{W}^{\mathrm{T}} \Phi r+\tilde{W}^{\mathrm{T}} \mathcal{L} \mathcal{O}+\frac{\tilde{W}^{\mathrm{T}} \mathcal{L} \mathcal{H O}}{\|\mathcal{O}\|}+\frac{1}{m_{s}}\|\dot{U}\|^{2} \\
& -\frac{1}{m_{s}}\left\|\dot{U}_{\tau}\right\|^{2}+\tau\|\dot{U}\|^{2}-\int_{t-\tau}^{t}\|\dot{U}(\lambda)\|^{2} d \lambda
\end{aligned}
$$

Consider the fact that the estimated FLS weights $\hat{W}$ are bounded by using the proposed adaptive law (31) with projection operation, hence as analyzed in [17], [18], one can deduce that the control $\dot{U}$ in (24) can be upper bounded by:

$$
\|\dot{U}\| \leq \beta+M\|r\|
$$

where $\beta$ and $M$ are positive constants.

Note that $\mathcal{O}=-\mathcal{H} \tilde{W}+\chi$ and $\lambda_{\min }(\mathcal{H})>\varrho>0$. Therefore, equation (42) can be further rewritten as:

$$
\begin{aligned}
\dot{V}_{2}= & -\tilde{W}^{\mathrm{T}} \Phi r+\tilde{W}^{\mathrm{T}} \mathcal{L}(-\mathcal{H} \tilde{W}+\chi)+\frac{\mathcal{L}\left(\chi^{\mathrm{T}}-\mathcal{O}^{\mathrm{T}}\right) \mathcal{O}}{\|\mathcal{O}\|} \\
& +\frac{1}{m_{s}}\|\dot{U}\|^{2}-\frac{1}{m_{s}}\left\|\dot{U}_{\tau}\right\|^{2}+\tau\|\dot{U}\|^{2}-\int_{t-\tau}^{t}\|\dot{U}(\lambda)\|^{2} d \lambda \\
\leq & -\tilde{W}^{\mathrm{T}} \Phi r-\underline{\zeta}(\mathcal{L}) \varrho\|\tilde{W}\|^{2}+\frac{1}{2} \bar{\zeta}(\mathcal{L})\left(\|\tilde{W}\|^{2}+\|\chi\|^{2}\right) \\
& +\bar{\zeta}(\mathcal{L})\|\chi\|+\frac{1}{m_{s}}(\beta+M\|r\|)^{2}-\frac{1}{m_{s}}\left\|\dot{U}_{\tau}\right\|^{2} \\
& +\tau(\beta+M\|r\|)^{2}-\int_{t-\tau}^{t}\|\dot{U}(\lambda)\|^{2} d \lambda \\
\leq & -\tilde{W}^{\mathrm{T}} \Phi r-\left(\underline{\zeta}(\mathcal{L}) \varrho-\frac{1}{2} \bar{\zeta}(\mathcal{L})\right) \| \tilde{W}^{2}+\mathcal{C}_{1} r^{2} \\
& -\frac{1}{m_{s}}\left\|\dot{U}_{\tau}\right\|^{2}-\int_{t-\tau}^{t}\|\dot{U}(\lambda)\|^{2} d \lambda+\beta^{2} \mathcal{C}_{1}+\mathcal{C}_{2}
\end{aligned}
$$

where $\mathcal{C}_{1}=\frac{1}{m_{s}}\left(1+\tau m_{s}\right)\left(1+M^{2}\right)$ and $\mathcal{C}_{2}=\frac{1}{2} \bar{\zeta}(\mathcal{L}) \bar{\chi}^{2}+$ $\bar{\zeta}(\mathcal{L}) \bar{\chi}$ are all positive consants. $\bar{\zeta}(\bullet)$ and $\underline{\zeta}(\bullet)$ represent the maximum and minimum singular value of the associated matrix.

By combining $\dot{V}_{1}$ given in (38) and $\dot{V}_{2}$ given in (44), we can obtain that

$$
\begin{aligned}
\dot{V}= & \dot{V}_{1}+\dot{V}_{2} \\
\leq & -\left(K-\frac{1}{2}\right) e_{1}^{2}-(\Lambda-1) S^{2}+\frac{1}{2}\left(1+\frac{1}{m_{s}}\right)\left\|e_{u}\right\|^{2} \\
& -\left(k_{s}+1-\mathcal{C}_{1}-\mathcal{C}_{3}\right) r^{2}-\left(\underline{\zeta}(\mathcal{L}) \varrho-\frac{1}{2} \bar{\zeta}(\mathcal{L})\right)\|\tilde{W}\|^{2} \\
& +\frac{1}{2} \zeta^{2}(\|z\|)\|z\|^{2}-\int_{t-\tau}^{t}\|\dot{U}(\lambda)\|^{2} d \lambda+\frac{1}{2} \varepsilon_{b}^{2} \\
& +\left(\mathcal{C}_{1}+\mathcal{C}_{3}\right) \beta^{2}+\mathcal{C}_{2}
\end{aligned}
$$

where $\mathcal{C}_{3}=\frac{1+M^{2}}{m_{s}}$ is also a positive constant.

Given the fact that $e_{u}=\frac{1}{m_{s}} \int_{t-\tau}^{t} \dot{U}(\lambda) d \lambda$ is true, hence the Cauchy-Schwarz inequality can be used to establish the following bound:

$$
\left\|e_{u}\right\|^{2} \leq \frac{\tau}{m_{s}^{2}} \int_{t-\tau}^{t}\|\dot{U}(\lambda)\|^{2} d \lambda
$$

In addition, the upper bound of $\mathcal{R}_{L K}$ can be obtained as:

$$
\begin{aligned}
\mathcal{R}_{L K} & \leq \tau \sup _{s \in(t-\tau, t)}\left(\int_{s}^{t}\|\dot{U}(\lambda)\|^{2} d s\right) \\
& \leq \tau \int_{t-\tau}^{t}\|\dot{U}(\lambda)\|^{2} d \lambda
\end{aligned}
$$

Hence, the following inequalities can be yielded by using (36a), (36b), (46) and (47):

$$
\begin{aligned}
& -\frac{1}{3} \int_{t-\tau}^{t}\|\dot{U}(\lambda)\|^{2} d \lambda \leq-\frac{m_{s}^{2}}{3 \tau}\left\|e_{u}\right\|^{2} \\
& -\frac{1}{3} \int_{t-\tau}^{t}\|\dot{U}(\lambda)\|^{2} d \lambda=-\frac{m_{s}}{3} \mathcal{P}_{L K} \\
& -\frac{1}{3} \int_{t-\tau}^{t}\|\dot{U}(\lambda)\|^{2} d \lambda \leq-\frac{1}{3 \tau} \mathcal{R}_{L K}
\end{aligned}
$$

Therefore, the bound of $\dot{V}$ can be further derived by using (48a)-(48c) as:

$$
\begin{aligned}
\dot{V} \leq & -\left(K-\frac{1}{2}\right) e_{1}^{2}-(\Lambda-1) S^{2}-\left(\frac{m_{s}^{2}}{3 \tau}-\frac{1}{2}\right. \\
& \left.-\frac{1}{2 m_{s}}\right)\left\|e_{u}\right\|^{2}-\left(k_{s}+1-\mathcal{C}_{1}-\mathcal{C}_{3}\right) r^{2}-(\underline{\zeta}(\mathcal{L}) \varrho \\
& \left.-\frac{1}{2} \bar{\zeta}(\mathcal{L})\right)\|\tilde{W}\|^{2}-\frac{m_{s}}{3} \mathcal{P}_{L K}-\frac{1}{3 \tau} \mathcal{R}_{L K} \\
& +\frac{1}{2} \zeta^{2}(\|z\|)\|z\|^{2}+\frac{1}{2} \varepsilon_{b}^{2}+\left(\mathcal{C}_{1}+\mathcal{C}_{3}\right) \beta^{2}+\mathcal{C}_{2} \\
\leq & -\left(\frac{\alpha}{2}-\frac{1}{2} \zeta^{2}(\|z\|)\right)\|z\|^{2}-\frac{\alpha}{2}\|z\|^{2} \\
& -\left(\underline{\zeta}(\mathcal{L}) \varrho-\frac{1}{2} \bar{\zeta}(\mathcal{L})\right) \| \tilde{W}^{2}-\frac{m_{s}}{3} \mathcal{P}_{L K}-\frac{1}{3 \tau} \mathcal{R}_{L K} \\
& +\frac{1}{2} \varepsilon_{b}^{2}+\left(\mathcal{C}_{1}+\mathcal{C}_{3}\right) \beta^{2}+\mathcal{C}_{2} \\
\leq & -\mathcal{A}\|\mathcal{Y}\|^{2}
\end{aligned}
$$

for any $\mathcal{Y}$ satisfying

$$
\|\mathcal{Y}\| \geq \sqrt{\frac{\varepsilon_{b}^{2}+2 \beta^{2}\left(\mathcal{C}_{1}+\mathcal{C}_{3}\right)+2 \mathcal{C}_{2}}{2}}
$$

where $\alpha, \mathcal{A}$ are defined by

$$
\begin{aligned}
& \alpha=\min \left\{\left(K-\frac{1}{2}\right),(\Lambda-1),\right. \\
& \left.\left(\frac{m_{s}^{2}}{3 \tau}-\frac{1}{2}-\frac{1}{2 m_{s}}\right),\left(k_{s}+1-\mathcal{C}_{1}-\mathcal{C}_{3}\right)\right\} \\
& \mathcal{A}=\frac{1}{2} \min \left\{\frac{\alpha}{2},\left(\underline{\zeta}(\mathcal{L}) \varrho-\frac{1}{2} \bar{\zeta}(\mathcal{L})\right), \frac{m_{s}}{3}, \frac{1}{3 \tau}\right\}
\end{aligned}
$$

According to the analysis given in [44] and the definition of Lyapunov function, we can conclude that $z, r, e_{1}, e_{u}, \tilde{W}$ 
and $U$ are all bounded. Moreover, one can verify from $\dot{V} \leq-\mathcal{A} V(\mathcal{Y})$ that $V(t) \leq V(0) e^{-\mathcal{A} t}$ is true, which implies that $V(t)$ and thus the vertical displacement $x_{1}$ will exponentially converge to a compact set defined by $\bar{\Omega}=$ $\left\{x_{1}(t)|| x_{1} \mid \leq \sqrt{\varepsilon_{b}^{2}+2 \beta^{2}\left(\mathcal{C}_{1}+\mathcal{C}_{3}\right)+2 \mathcal{C}_{2}}\right\}$.

Remark 5: The PE condition is necessary to guarantee the parameter estimation convergence, which has been well-known in the system identification field. For the studied vehicle suspension system (1), the PE condition is generally satisfied because of the continuously injected road disturbance $z_{r}$ from the irregular road roughness. Moreover, the online validation of the PE condition is usually a challenging task, which also remains as an open problem. Based on Lemma 2, an intuitive approach is suggested by numerically calculating the minimum eigenvalue of the designed auxiliary matrix $\mathcal{H}$ and then testing for the condition $\lambda_{\min }(\mathcal{H})>0$, which can be conducted online feasibly.

Remark 6: As shown in the above proof, we introduce Lyapunov-Krasovskii functionals $\mathcal{P}_{L K}, \mathcal{R}_{L K}$ as inspired by [16] to address the effect of input delay $\tau$, such that the stability of the proposed closed-loop system with the controller given in (23) can be strictly guaranteed. Moreover, apart from the performance requirement PR1 as guaranteed in Theorem 1 , the analysis of the other two performance requirements follows a similar procedure given in [7], which consists of two steps: 1) prove the boundedness of $x_{3}, x_{4}$ based on Lyapunov theory and the boundedness of system signals; 2 ) calculate and quantify the bounds of dynamic tire and suspension stroke. Due to the page limit, we refer to [7] for the detailed analysis.

\section{Finite-Time Parameter Convergence}

In this subsection, we will further prove the finite-time convergence of the estimated FLS weights $\hat{W}$ with adaptive law given in (31) and (32). The main result of this subsection is given as follows:

Theorem 2: The estimation error of the FLS weights $\tilde{W}$ with adaptive law given in (31) and (32) will converge to a small set around zero (i.e. $\tilde{W}=\mathcal{H}^{-1} \chi$ ) in finite-time.

Proof: We first investigate the time derivative of $\mathcal{H}^{-1} \mathcal{O}$. Given the fact that $\mathcal{H}^{-1} \mathcal{O}=-\tilde{W}+\mathcal{H}^{-1} \chi$, one can obtain:

$$
\frac{\partial \mathcal{H}^{-1} \mathcal{O}}{\partial t}=-\dot{\tilde{W}}+\frac{\partial \mathcal{H}^{-1}}{\partial t} \chi+\mathcal{H}^{-1} \dot{\chi}=\dot{\hat{W}}+\chi^{\prime}
$$

where $\chi^{\prime}=-\mathcal{H}^{-1} \dot{\mathcal{H}} \mathcal{H}^{-1} \chi+\mathcal{H}^{-1} \dot{\chi}$ is a bounded variable, which can be verified by considering the fact that the regressor matrix $\mathcal{H}$ and the residual FLS error $\chi$ are all bounded.

Select the Lyapunov function $V_{3}$ as:

$$
V_{3}=\frac{1}{2} \mathcal{O}^{\mathrm{T}} \mathcal{H}^{-1} \mathcal{H}^{-1} \mathcal{O}
$$

Recalling (30)-(32) together with (53), the time derivative of $V_{3}$ can be obtained as:

$$
\begin{aligned}
\dot{V}_{3}= & \mathcal{O}^{\mathrm{T}} \mathcal{H}^{-1} \frac{\partial \mathcal{H}^{-1} \mathcal{O}}{\partial t}=\mathcal{O}^{\mathrm{T}} \mathcal{H}^{-1} \Gamma\left((\Phi r-\mathcal{M})+\chi^{\prime}\right) \\
= & \mathcal{O}^{\mathrm{T}} \mathcal{H}^{-1} \Gamma \Phi r-\mathcal{O}^{\mathrm{T}} \mathcal{H}^{-1} \Gamma \mathcal{L} \mathcal{O} \\
& -\Gamma \mathcal{L}\|\mathcal{O}\|+\mathcal{O}^{\mathrm{T}} \mathcal{H}^{-1} \Gamma \chi^{\prime} \\
\leq & -\left[\underline{\zeta}(\Gamma) \underline{\zeta}(\mathcal{L})-\bar{\zeta}(\Gamma)\left\|\mathcal{H}^{-1}\right\|\|\Phi\|\|r\|\right. \\
& \left.-\bar{\zeta}(\Gamma)\left\|\mathcal{H}^{-1} \chi^{\prime}\right\|\right]\|\mathcal{O}\| \\
\leq & -\frac{\sqrt{2}}{\bar{\zeta}\left(\mathcal{H}^{-1}\right)}\left[\underline{\zeta}(\Gamma) \underline{\zeta}(\mathcal{L})-\bar{\zeta}(\Gamma) \bar{\zeta}\left(\mathcal{H}^{-1}\right)\|\Phi\|\|r\|\right. \\
& \left.-\bar{\zeta}(\Gamma) \bar{\zeta}\left(\mathcal{H}^{-1}\right)\left\|\chi^{\prime}\right\|\right] \sqrt{V_{3}}
\end{aligned}
$$

Because $r$ will converge to a small compact set as proved in Theorem 1 and the FLS regressor vector $\Phi$ is bounded, then there exists a time $T_{1}$ and large learning gain $\mathcal{L}$, such that for all $t>T_{1}$ it can be claimed that

$$
\underline{\zeta}(\Gamma) \underline{\zeta}(\mathcal{L})>\bar{\zeta}(\Gamma) \bar{\zeta}\left(\mathcal{H}^{-1}\right)\left(\|\Phi\|\|r\|+\left\|\chi^{\prime}\right\|\right)
$$

Thus, there is a time instant $T_{2}$ and a constant $\epsilon>0$, such that the condition $\dot{V}_{3} \leq-\epsilon \sqrt{V_{3}}$ is true for all $t>T_{2}$. Therefore, based on the stability analysis of sliding mode control in [45], the finite-time convergence of the estimation error $\mathcal{H}^{-1} \mathcal{O}$ to zero can be claimed. Consequently, by recalling the fact $\mathcal{H}^{-1} \mathcal{O}=-\tilde{W}+\mathcal{H}^{-1} \chi$, the FLS weights error $\tilde{W}$ will converge to a small compact set defined by $\mathcal{H}^{-1} \chi$, of which the size depends on the excitation level (e.g. the amplitude of $\mathcal{H}^{-1}$ ), and the FLS inherent error $\varepsilon$ leading to $\chi$. Thus the finite-time parameter estimation performance can be achieved. This completes the proof.

Remark 7: The main aim of the current study is to propose a new compensator to address the input delay in the active suspension systems, which can be incorporated into adaptive fuzzy control design with guaranteed convergence and thus enhanced suspension response. Moreover, it is noted that different actuators (e.g. electrical motors, hydraulic cylinder) can be used in the active suspension systems. Hence, the dynamics of actuator used to generate the required force are not considered explicitly in this paper.

\section{Simulation Studies}

In this section, we provide numerical simulation results to exemplify the proposed control method in terms of suspension displacement response and ride comfort. To cover more realistic applications, a professional simulation software for vehicle systems, Carsim (Version 8.1), is employed to generate realistic driving road conditions collected from the realistic experimental data, which are then used to build a combined dynamic simulator together with Matlab/Simulink.

The unknown and nonlinear dynamics of the spring, damper and tire are chosen as described as [5]:

$$
\begin{aligned}
& F_{d}\left(x_{2}, x_{4}\right)=b_{s}\left(x_{2}-x_{4}\right) \\
& F_{s}\left(x_{1}, x_{3}\right)=k_{s}\left(x_{1}-x_{3}\right)+k_{s n}\left(x_{1}-x_{3}\right)^{3} \\
& F_{t}\left(x_{3}, z_{r}\right)=k_{t}\left(x_{3}-z_{r}\right), F_{b}\left(x_{4}, \dot{z}_{r}\right)=b_{t}\left(x_{4}-\dot{z}_{r}\right)
\end{aligned}
$$

where $b_{s}$ denotes the damping coefficient of the suspension components; $k_{s}$ and $k_{s n}$ represent the stiffening coefficients of 
the spring; $k_{t}$ and $b_{t}$ are the stiffness and damping coefficients of the tire. The parameters of the quarter-car model shown in Fig.1 and (56) are chosen as [5]: $m_{s}=600 \mathrm{~kg}, m_{u}=$ $60 \mathrm{~kg}, b_{s}=2500 \mathrm{Ns} / \mathrm{m}, k_{s}=18000 \mathrm{~N} / \mathrm{m}, k_{s n}=$ $1000 \mathrm{~N} / \mathrm{m}, k_{t}=200000 \mathrm{~N} / \mathrm{m}, b_{t}=1000 \mathrm{~N} / \mathrm{m}$ and the control input time delay is $\tau=40 \mathrm{~ms}$.

The two riding road conditions generated from Carsim are simulated: a) bumpy and random road condition; b) straight lane with example road roughness, whose corresponding profiles are given in Fig.4. Note that the adopted road condition a) in this paper has more realistic dynamic excitations, and is different with the widely used ones in the existing literatures, e.g. [3], [5], [8], [10]. Those previous adopted road elevations have neither consistent shocks nor vibrations, but only a bump signal appearing in the first few seconds is simulated. The initial system conditions are chosen as $x_{1}(0)=0.06$ and $x_{i}(0)=0, i=2,3,4$. For the FLS used to accommodate the unknown nonlinearities, we choose $-4,-3,3,4$ as the central points and 80 as the stand derivations of regressor function, and the initial FLS weights are chosen as $\hat{W}(0)=0$. The unknown FLS weights are then updated by the designed FT parameter estimation algorithm (31). The control gains are chosen as $K=10, \Lambda=10, k_{s}=100$. The parameters of filter operation are set as $\kappa=0.001, \ell=1$ and the adaptive gains are $\Gamma=30 \operatorname{diag}[I]_{64 \times 64}$ and $\mathcal{L}=0.01$ diag $[I]_{64 \times 64}$ in the road condition a). In road $b$ ), the adaptive gains are slightly modified to achieve better desired convergence, i.e. $\Gamma=80 \operatorname{diag}[I]_{64 \times 64}$ and $\mathcal{L}=0.06 \operatorname{diag}[I]_{64 \times 64}$, while the other parameters in the FLS, filter operation and control gains are the same as used for the road condition a).

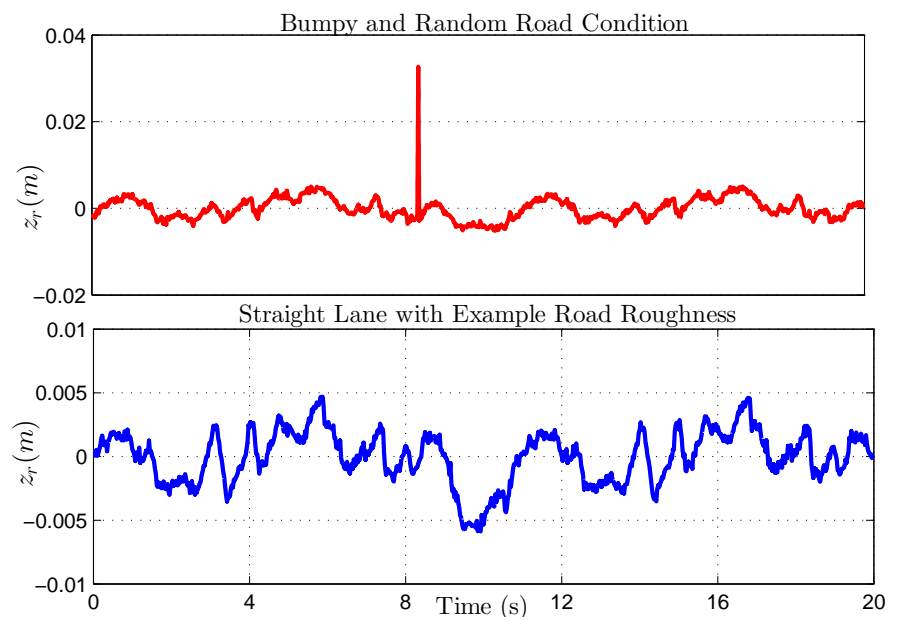

Fig. 4. Ground road elevation of two tested road conditions.

Figs. 5-7 show the comparative simulation results of vehicle motion displacements and other suspension performance requirements. Fig. 5 provides the profiles of vertical displacements with different control approaches. One can find from Fig.5 that the proposed control with both the FT adaptive law and the traditional $e$-modification method is able to isolate the uninterrupted vibrations/shocks transmitted from the irregular road roughness effectively, and maintain the stability of the vehicle body. In particular, less fluctuations and lower peaks are obtained compared with the passive suspension systems. Moreover, from the bottom subfigure of Fig.5, one can find that the proposed control with new FT adaptive learning scheme can provide superior capability to regulate the vertical displacement around zero over the traditional $e$-modification method. This is attributed to the faster and smoother convergence of the FLS weights derived by using the proposed FT algorithm (31). The acceleration responses of the vehicle body under two different riding road conditions are given in Fig.6. As shown in Fig.6, it is obvious that the magnitude of the vehicle body acceleration can be greatly reduced with the proposed adaptation in comparison with the $e$-modification method. In this sense, the proposed new learning algorithm can also help to provide better ride comfort, i.e. smaller acceleration values.

The results of other vehicle performance requirements (i.e. PR2 and PR3) under the given road condition b) are also plotted in Fig.7. It can be found from the top subfigure of Fig.7 that the dynamic tire loads are all guaranteed within the limitation $0.1 \mathrm{~m}$ with the two different methods. The bottom subfigure of Fig.7 shows the suspension stroke responses, which indicates that the suspension movements with the two controllers are all strictly limited within the allowable maximum $z_{\max }=0.15 \mathrm{~m}$. However, it is evident that the new FT adaptation algorithm suggested in this paper provides smaller tire deflections and faster convergence responses, which implies that this new learning approach has better ability in ensuring the driving safety for drivers and/or occupants. Consequently, we can conclude that although the two control methods can ensure the required suspension performance (i.e. PR1, PR2 and PR3), the suggested new learning algorithm has superiority over conventional learning method, thereby improving the overall active suspension performance. Similar simulation results and conclusions can be observed in bumpy and random road conditions, which are not presented again.

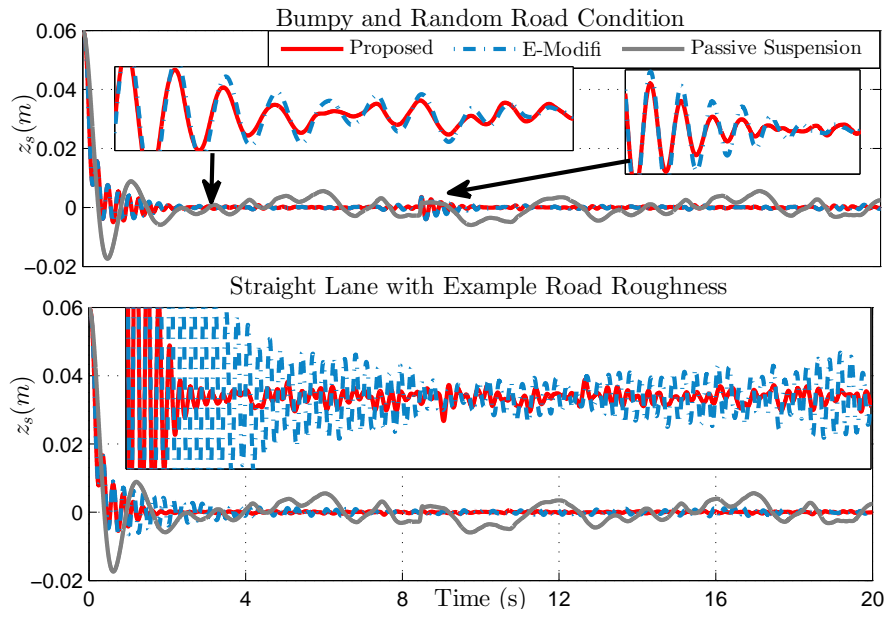

Fig. 5. Vehicle displacement under different road conditions.

To demonstrate the efficacy of the proposed FT parameter estimation algorithm (31) more concretely, the profiles of the estimated FLS weights under the road condition b) are depicted in Fig.8. The convergence response of the FLS weights 

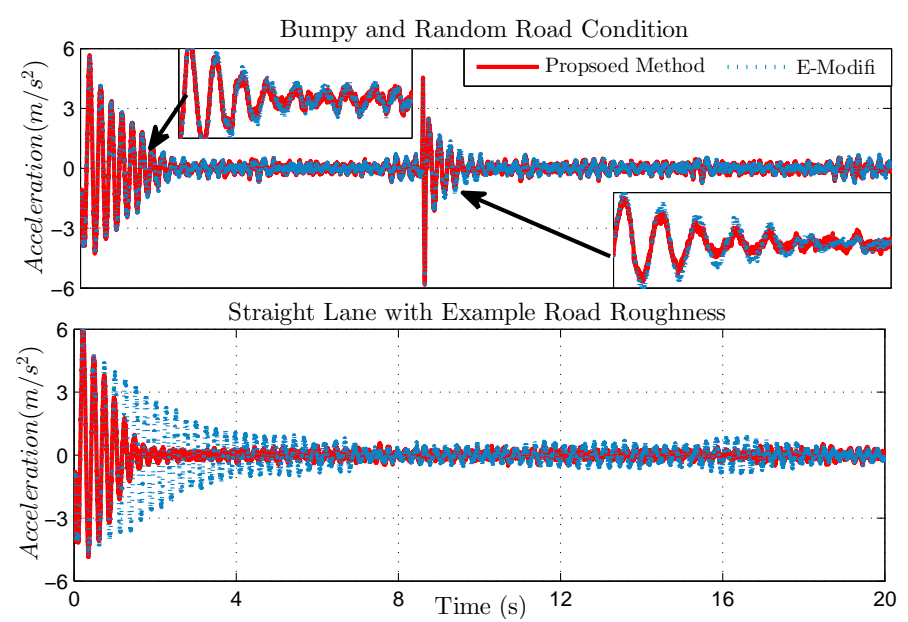

Fig. 6. Comparative results of acceleration responses.

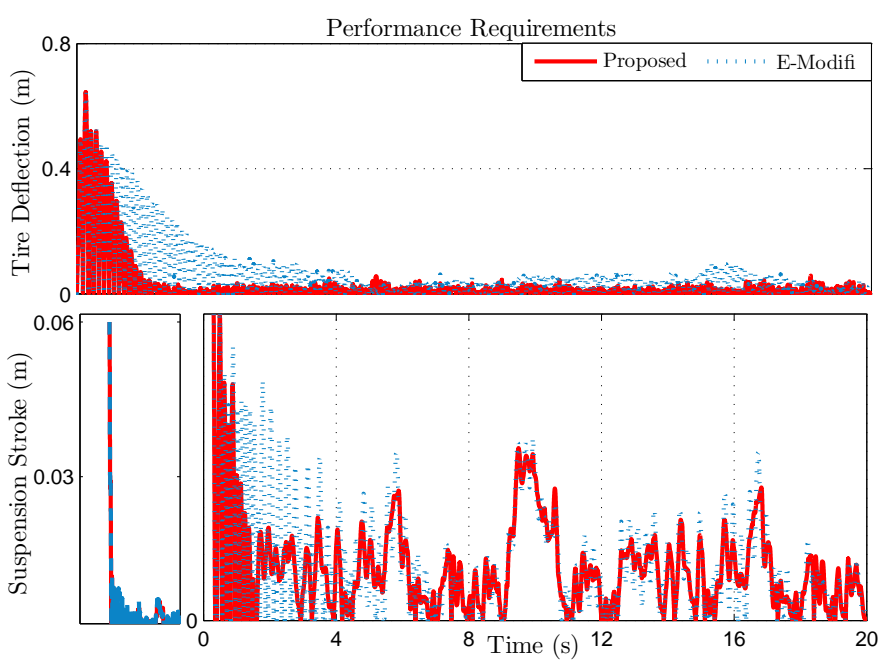

Fig. 7. Comparative results of suspension performance requirements.

updated with the proposed FT algorithm (31) is provided in the top subfigure of Fig.8, and the estimated FLS weights by using the $e$-modification method is given in the bottom subfigure of Fig.8. We can observe from Fig.8 that the FLS weights $\hat{W}$ are convergent with a faster rate and smoother steady-state response by using the proposed FT method (31), while the FLS weights $\hat{W}$ with $e$-modification based learning algorithm can hardly achieve convergence though they are bounded. Hence, we can conclude that the convergence of FLS weights can be significantly improved via the new parameter estimation algorithm, which in turn contributes to improving the control performance.

Finally, the effectiveness of the developed input delay compensation strategy with (14) is shown in Figs. 9-10. The responses of vehicle displacement under different input delay values are presented in Fig.9, without using the input delay compensation (we can set $e_{u}=0$ in (15)). We can see that with the increase of input delay value (i.e. $\tau=10 \mathrm{~ms}, 20 \mathrm{~ms}, 30 \mathrm{~ms}, 40 \mathrm{~ms}$ ), the control without $e_{u}$ gradually looses the ability to regulate the vertical motion

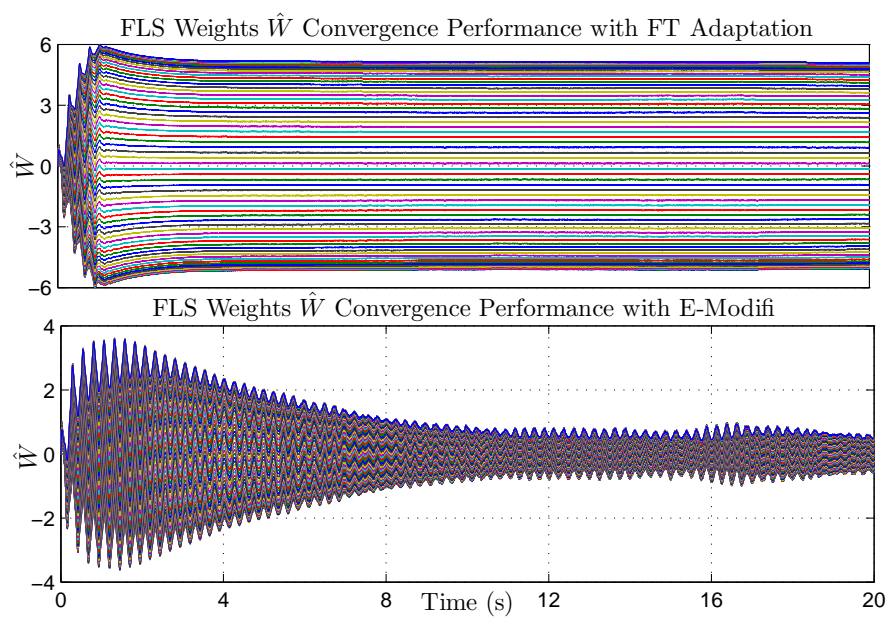

Fig. 8. Profiles of FLS weights $\hat{W}$ with different adaptations.

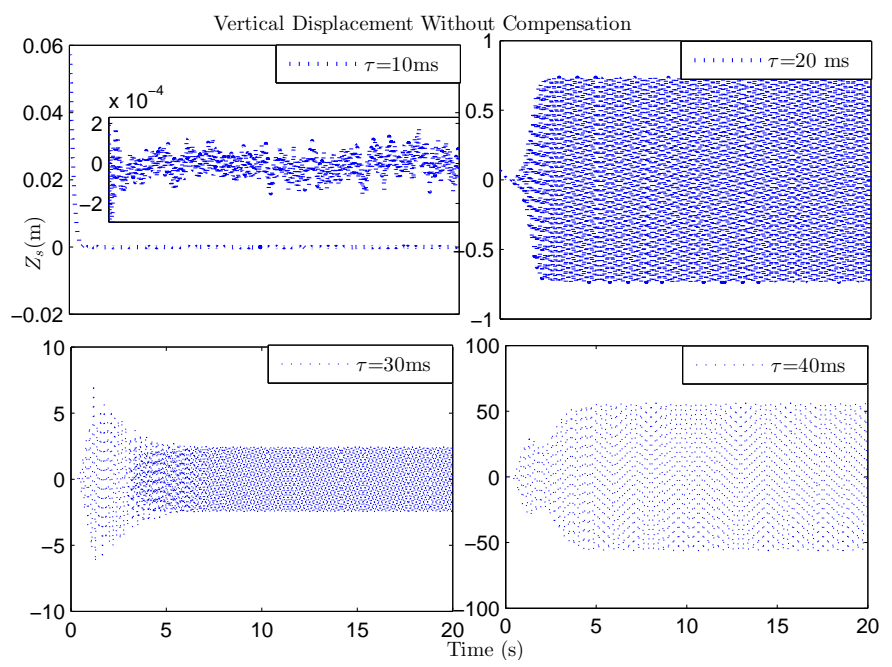

Fig. 9. Vehicle displacement without input delay compensation.
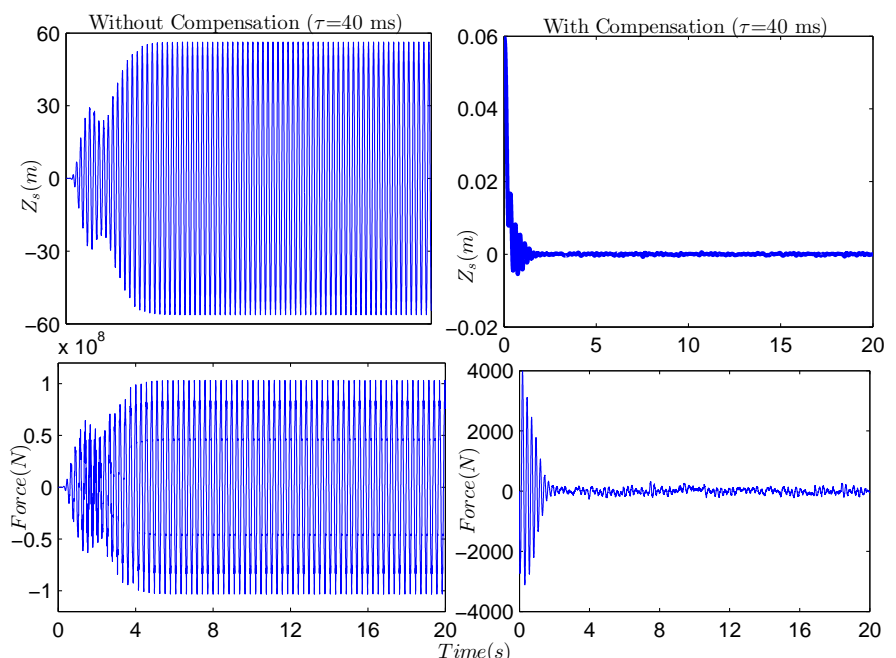

Fig. 10. Displacement and control signal with/without compensation for $\tau=40 \mathrm{~ms}$. 
displacement, and thus fails to strictly ensure the ride comfort and driving safety due to the exaggerated vehicle motion. In Fig.10, comparative results regarding the vertical suspension displacement $x_{1}$ and its corresponding control input force $U$ with/without the input delay compensation $e_{u}$ are provided. As it is shown in Fig.10, with the help of the proposed delay compensation strategy, not only the vertical displacement $x_{1}$ can be regulated within a small region around zero (i.e. $x_{1} \rightarrow 0$ ), but also the required control force can be retained within a feasible bound. Thus, the proposed control actions with delay compensator can be implemented by practical actuators. From these comparative results, we can claim that the proposed control method with FT learning algorithm and input delay compensator can effectively handle the effect of input delay and unknown dynamics, thereby making the active suspension systems work safely and reliably.

\section{CONCLUSION}

In this paper, we proposed a new adaptive fuzzy control approach for uncertain nonlinear active suspension systems subject to input time delay and unknown nonlinear dynamics. An appropriate input delay compensation strategy is first introduced to obtain a delay-free term to eliminate the effect of input delay in the control system. Then an FLS was used as the function approximation, and incorporated into the adaptive control design, such that the uncertain dynamics and unknown nonlinearities can be addressed. Unlike existing conventional adaptive control designs, a novel finite-time parameter estimation algorithm driven by the parameter estimation error was developed to online update the FLS weights, such that the estimated FLS weights converge to a small neighbourhood around their ideal values in finite-time. The closed-loop system stability analysis was carried out based on the LyapunovKrasovskii functional. Simulation results in terms of a dynamic simulator consisting of a commercial software, Carsim, and Matlab illustrated that the proposed control method can handle both the input delay and uncertainties, and the new learning method suggested in this paper also obtained superior performance in terms of both control and parameter estimation responses. Future work will focus on exploiting the proposed adaptive control for active suspension systems subject to timevarying input delay and unknown actuator dynamics.

\section{REFERENCES}

[1] D. Cao, X. Song, and M. Ahmadian, "Editors perspectives: road vehicle suspension design, dynamics, and control," Veh. Syst. Dyn., vol. 49, no. 1-2, pp. 3-28, 2011.

[2] D. Hrovat, "Survey of advanced suspension developments and related optimal control applications1, 2," Automatica, vol. 33, no. 10, pp. 1781$1817,1997$.

[3] H. Li, J. Yu, C. Hilton, and H. Liu, "Adaptive sliding-mode control for nonlinear active suspension vehicle systems using T-S fuzzy approach," IEEE Trans. Ind. Electron., vol. 60, no. 8, pp. 3328-3338, 2013.

[4] W.-Y. Wang, M.-C. Chen, and S.-F. Su, "Hierarchical T-S fuzzy-neural control of anti-lock braking system and active suspension in a vehicle," Automatica, vol. 48, no. 8, pp. 1698-1706, 2012.

[5] W. Sun, J. Li, Y. Zhao, and H. Gao, "Vibration control for active seat suspension systems via dynamic output feedback with limited frequency characteristic," Mechatronics, vol. 21, no. 1, pp. 250-260, 2011.

[6] Y. Jia, "Robust control with decoupling performance for steering and traction of 4ws vehicles under velocity-varying motion," IEEE Trans. Control Syst. Technol., vol. 8, no. 3, pp. 554-569, 2000.
[7] Y. Huang, J. Na, X. Wu, and G. Gao, "Approximation-free control for vehicle active suspensions with hydraulic actuator," IEEE Trans. Ind. Electron., vol. 65, no. 9, pp. 7258-7267, 2018.

[8] Y. Huang, J. Na, X. Wu, X. Liu, and Y. Guo, "Adaptive control of nonlinear uncertain active suspension systems with prescribed performance," ISA Trans., vol. 54, pp. 145-155, 2015.

[9] W. Sun, Z. Zhao, and H. Gao, "Saturated adaptive robust control for active suspension systems," IEEE Trans. Ind. Electron., vol. 60, no. 9, pp. 3889-3896, 2013.

[10] H. Li, X. Jing, H.-K. Lam, and P. Shi, "Fuzzy sampled-data control for uncertain vehicle suspension systems," IEEE Trans. Cybern., vol. 44, no. 7, pp. 1111-1126, 2014.

[11] H. Li, X. Jing, and H. R. Karimi, "Output-feedback-based $H_{\infty}$ control for vehicle suspension systems with control delay," IEEE Trans. Ind. Electron., vol. 61, no. 1, pp. 436-446, 2014.

[12] O. J. Smith, "A controller to overcome dead time," ISA J., vol. 6, pp 28-33, 1959.

[13] K. J. Astrom, C. C. Hang, and B. Lim, "A new smith predictor for controlling a process with an integrator and long dead-time," IEEE Trans. Autom. Control., vol. 39, no. 2, pp. 343-345, 1994.

[14] Y. Yildiz, A. M. Annaswamy, D. Yanakiev, and I. Kolmanovsky, "Sparkignition-engine idle speed control: An adaptive control approach," IEEE Trans. Control Syst. Technol., vol. 19, no. 5, pp. 990-1002, 2011.

[15] H. Li, L. Wang, H. Du, and A. Boulkroune, "Adaptive fuzzy backstepping tracking control for strict-feedback systems with input delay," IEEE Trans. Fuzzy Syst., vol. 25, no. 3, pp. 642-652, 2017.

[16] N. Fischer, A. Dani, N. Sharma, and W. E. Dixon, "Saturated control of an uncertain nonlinear system with input delay," Automatica, vol. 49, no. 6, pp. 1741-1747, 2013.

[17] S. Obuz, J. R. Klotz, R. Kamalapurkar, and W. Dixon, "Unknown time-varying input delay compensation for uncertain nonlinear systems," Automatica, vol. 76, pp. 222-229, 2017.

[18] R. Kamalapurkar, N. Fischer, S. Obuz, and W. E. Dixon, "Time-varying input and state delay compensation for uncertain nonlinear systems," IEEE Trans. Autom. Control., vol. 61, no. 3, pp. 834-839, 2016.

[19] C. Yang, C. Chen, W. He, R. Cui, and Z. Li, "Robot learning system based on adaptive neural control and dynamic movement primitives," IEEE Trans. Neural Netw. Learn. Syst., no. 99, pp. 1-11, 2018.

[20] Y.-J. Liu, S. Tong, C. P. Chen, and D.-J. Li, "Neural controller designbased adaptive control for nonlinear mimo systems with unknown hysteresis inputs," IEEE Trans. Cybern., vol. 46, no. 1, pp. 9-19, 2016.

[21] H. Li, S. Zhao, W. He, and R. Lu, "Adaptive finite-time tracking control of full state constrained nonlinear systems with dead-zone," Automatica, vol. 100, pp. 99-107, 2019.

[22] Y.-J. Liu, Y. Gao, S. Tong, and Y. Li, "Fuzzy approximation-based adaptive backstepping optimal control for a class of nonlinear discretetime systems with dead-zone," IEEE Trans. Fuzzy Syst., vol. 24, no. 1, pp. 16-28, 2016.

[23] Y. Li and S. Tong, "Adaptive fuzzy output constrained control design for multi-input multioutput stochastic nonstrict-feedback nonlinear systems," IEEE Trans. Cybern., vol. 47, no. 12, pp. 4086-4095, 2017.

[24] Y.-J. Liu and S. Tong, "Adaptive NN tracking control of uncertain nonlinear discrete-time systems with nonaffine dead-zone input," IEEE Trans. Cybern., vol. 45, no. 3, pp. 497-505, 2015.

[25] N. Wang, M. J. Er, J.-C. Sun, and Y.-C. Liu, "Adaptive robust online constructive fuzzy control of a complex surface vehicle system," IEEE Trans. Cybern., vol. 46, no. 7, pp. 1511-1523, 2016.

[26] Q. Chen, S. Xie, M. Sun, and X. He, "Adaptive non-singular fixed-time attitude stabilization of uncertain spacecraft," IEEE Tran. Aero. Electron. Syst., vol. 54, no. 6, pp. 2937-2950, 2018.

[27] S. Wang, H. Yu, J. Yu, J. Na, and X. Ren, "Neural-network-based adaptive funnel control for servo mechanisms with unknown dead-zone," IEEE Tran. cybern., vol. pp, no. 99, pp. 1-12, 2018.

[28] N. Wang, S.-F. Su, J. Yin, Z. Zheng, and M. J. Er, "Global asymptotic model-free trajectory-independent tracking control of an uncertain marine vehicle: an adaptive universe-based fuzzy control approach," IEEE Trans. Fuzzy Syst., vol. 26, no. 3, pp. 1613-1625, 2018.

[29] Y.-C. Hsueh, S.-F. Su, and M.-C. Chen, "Decomposed fuzzy systems and their application in direct adaptive fuzzy control," IEEE Trans. Cybern., vol. 44, no. 10, pp. 1772-1783, 2014.

[30] T. Tao and S.-F. Su, "Moment adaptive fuzzy control and residue compensation." IEEE Trans. Fuzzy Syst., vol. 22, no. 4, pp. 803-816, 2014.

[31] Y.-J. Liu, W. Wang, S.-C. Tong, and Y.-S. Liu, "Robust adaptive tracking control for nonlinear systems based on bounds of fuzzy approximation parameters," IEEE Trans. Syst., Man, Cybern. A, Syst., Humans, vol. 40, no. 1, pp. 170-184, 2010. 
[32] H. Ma, H. Liang, Q. Zhou, and C. K. Ahn, "Adaptive dynamic surface control design for uncertain nonlinear strict-feedback systems with unknown control direction and disturbances," IEEE Tran. Syst., Man, Cybern., Syst., no. 99, pp. 1-10, 2018.

[33] L. Cao, H. Li, and Q. Zhou, "Adaptive intelligent control for nonlinear strict-feedback systems with virtual control coefficients and uncertain disturbances based on event-triggered mechanism," IEEE Tran. cybern., vol. 48, no. 12, pp. 3390-3402, 2018.

[34] J.-J. E. Slotine, W. Li et al., Applied nonlinear control. Prentice hall Englewood Cliffs, NJ, 1991, vol. 199, no. 1.

[35] V. Adetola and M. Guay, "Finite-time parameter estimation in adaptive control of nonlinear systems," IEEE Trans. Autom. Control., vol. 53, no. 3, pp. 807-811, 2008 .

[36] J. Na, M. N. Mahyuddin, G. Herrmann, X. Ren, and P. Barber, "Robust adaptive finite-time parameter estimation and control for robotic systems," Int. J. Robust Nonlin. Control., vol. 25, no. 16, pp. 3045-3071, 2015.

[37] J. Na, Y. Huang, X. Wu, G. Gao, G. Herrmann, and J. Z. Jiang, "Active adaptive estimation and control for vehicle suspensions with prescribed performance," IEEE Trans. Control Syst. Technol., vol. 26, no. 6, pp. 2063-2077, 2018.

[38] J. Na, A. S. Chen, G. Herrmann, R. Burke, and C. Brace, "Vehicle engine torque estimation via unknown input observer and adaptive parameter estimation," IEEE Tran. Veh. Technol., vol. 67, no. 1, pp. 409-422, 2018.

[39] L.-X. Wang and J. M. Mendel, "Fuzzy basis functions, universal approximation, and orthogonal least-squares learning," IEEE Trans. Neural Netw., vol. 3, no. 5, pp. 807-814, 1992.

[40] S. Wang, J. Na, and X. Ren, "Rise-based asymptotic prescribed performance tracking control of nonlinear servo mechanisms," IEEE Tran. Syst., Man, Cybern., Syst., vol. PP, no. 99, pp. 1-12, 2017.

[41] Q. Yang, S. Jagannathan, and Y. Sun, "Robust integral of neural network and error sign control of mimo nonlinear systems," IEEE Trans. Neural Netw. Learn. Syst., vol. 26, no. 12, pp. 3278-3286, 2015.

[42] R. Kamalapurkar, J. A. Rosenfeld, J. Klotz, R. J. Downey, and W. E. Dixon, "Supporting lemmas for rise-based control methods," arXiv preprint arXiv:1306.3432, 2013.

[43] J. Na, G. Herrmann, and K. Zhang, "Improving transient performance of adaptive control via a modified reference model and novel adaptation," Int. J. Robust Nonlin. Control., vol. 27, no. 8, pp. 1351-1372, 2017.

[44] H. K. Khalil, "Noninear systems," Prentice-Hall, New Jersey, vol. 2, no. 5, pp. 5-1, 1996.

[45] S. P. Bhat and D. S. Bernstein, "Continuous finite-time stabilization of the translational and rotational double integrators," IEEE Trans. Autom. Control., vol. 43, no. 5, pp. 678-682, 1998.

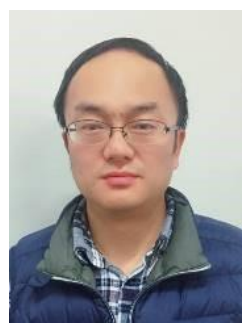

Jing Na (M'15) received the B.Eng. and $\mathrm{Ph} . \mathrm{D}$. degrees in control engineering from the School of Automation, Beijing Institute of Technology, Beijing, China, in 2004 and 2010, respectively.

From 2011 to 2013, he was a Monaco/ITER Postdoctoral Fellow with the ITER Organization, Saint-Paul-lès, Durance, France. From 2015 to 2017 he was a Marie Curie Intra-European Fellow with the Department of Mechanical Engineering, University of Bristol, U.K. Since 2010, he has been with the Faculty of Mechanical and Electrical Engineering, Kunming University of Science and Technology, Kunming, China, where he became a Full Professor in 2013. He has coauthored one monograph published in Elsevier and authored or co-authored more than 100 international journal and conference papers. His research interests include intelligent control, adaptive parameter estimation, nonlinear control and applications for robotics, vehicle systems and wave energy convertor, etc.

Dr Na is currently an Associate Editor of Neurocomputing and has served as the IPC Chair of ICMIC 2017. He was the recipient of a Marie Curie Fellowship from EU, the Best Application Paper Award of the 3rd IFAC International Conference on Intelligent Control and Automation Science (IFAC ICONS 2013), and the 2017 Hsue-shen Tsien Paper Award.

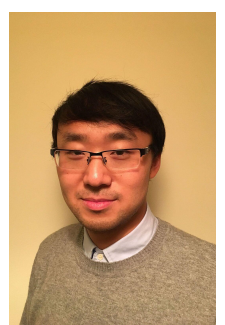

Yingbo Huang (StM'18) received the B.S. degree from Lanzhou City University, China, in 2013. He is currently pursuing the Ph.D degree with the Faculty of Mechanical and Electrical Engineering, Kunming University of Science and Technology, China.

His current research interests include adaptive control and transient performance improvement of nonlinear systesms with application to vehicle suspension systems.

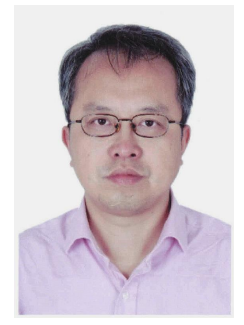

Xing Wu received the B.Sc. and M.Sc. degrees from Kunming University of Science and Technology, China, in 1994 and 1997, respectively, and the Ph.D. degree in mechanical engineering from Shanghai Jiao Tong University, China, in 2005.

$\mathrm{He}$ is currently a Professor with the Faculty of Mechanical and Electrical Engineering, Kunming University of Science and Technology. His current research interests include modern signal processing theory and their applications on fault feature extracting, and internet-based mechanical fault diagnosis technology and expert systems.

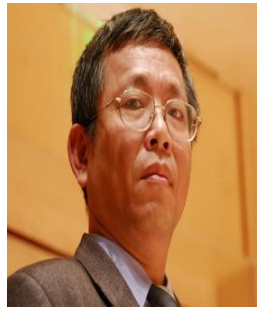

Shun-Feng Su (S'89-M'91-SM'05-F'10) received the B.S. degree in electrical engineering from National Taiwan University, Taipei, Taiwan, in 1983 and the M.S. and Ph.D. degrees in electrical engineering from Purdue University, West Lafayette, IN, USA, in 1989 and 1991, respectively.

He is currently a Chair Professor with the Department of Electrical Engineering, National Taiwan University of Science and Technology, Taipei. He has published more than 300 refereed journal and conference papers in the areas of robotics, intelligent control, fuzzy systems, neural networks, and nonderivative optimization. His current research interests include computational intelligence, machine learning, virtual reality, intelligent transportation systems, smart home, robotics, and intelligent control.

$\mathrm{Dr}$. Su is very active in various international/domestic professional societies. $\mathrm{He}$ is the Past President of the International Fuzzy Systems Association. He also serves as a Board Member of various academic societies. He also acted as the General Chair, the Program Chair, or various positions for several international and domestic conferences. He currently serves as an Associate Editors for the IEEE TRANSACTIONS ON CYBERNETICS, the IEEE/CAA Journal Automatica Sinca, and the IEEE ACCESS, a Subject Editor (Electrical Engineering) for the Journal of the Chinese Institute of Engineers, and the Editor-in-Chief of the International Journal of Fuzzy Systems. He is also the CACS Fellow.

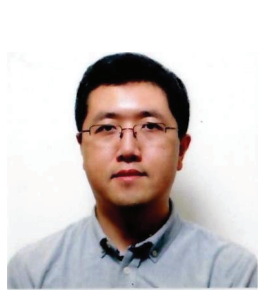

Guang Li (M'09) received his Ph.D. degree in Electrical and Electronics Engineering, specialized in control systems, from the University of Manchester, in 2007. He is currently a senior lecturer in dynamics modelling and control in Queen Mary University of London, UK.

His current research interests include constrained optimal control, model predictive control, adaptive robust control and control applications including renewable energies and energy storage, etc. 